\title{
Cross Layer Based Cooperative Spectrum Sensing for Cognitive Radio
}

\section{Networks}

\author{
G.A.Pethunachiyar ${ }^{1}$, and B.Sankaragomathi ${ }^{2}$ \\ ${ }^{1}$ Department of Computer Applications, The Tamilnadu Dr.Ambedkar Law University, Chennai,Tamilnadu, India. \\ ${ }^{2}$ Department of Electronics and Instrumentation Engineering, National Engineering College, Kovilpatti,Tamilnadu, India.
}

ORCID: 0000-0001-9649-7100 (Pethunachiyar)

\begin{abstract}
The Cognitive Radio(CR) is an intelligent and efficient technology needed in today's wireless environment to make use of the underutilized spectrum resources.The $\mathrm{CR}$ users have to continuously sense the spectrum before accessing to avoid the interference with the primary user. Spectrum sensing is primarily a physical layer function. Sharing the sensing information is needed for the cooperative detection. It necessitates a cross layer design between spectrum sharing and spectrum sensing. The cross layer design approach is proposed by reviewing the performance of the energy detection and Orthogonal Frequency Division Multiplexing(OFDM)Sensing technique.Also,the more number of $\mathrm{CR}$ users are involved to analyze the results of the cooperative spectrum sensing. Finally,the probability of detection and the probability of false alarm for the different number of samples and signal to noise ratio values are analyzed and presented for the proposed framework
\end{abstract}

Keywords:Cognitive Radio, Cross Layer,Energy

Detection,Primary User,Spectrum

\section{INTRODUCTION}

Spectrum in the licensed bandwidth is not utilized all the time [1]. Due to the increase in the usage of wireless networks, the scarcity of spectrum arises. According to Federal Communication Commission (FCC), the licensed bandwidth can be used by the unlicensed user when it is not used by the primary user (PU). The primary user is the user who gets the license for using the corresponding spectrum. The unlicensed user known as the secondary user(SU) can use PU spectrum without affecting the primary user activities when it is available for usage. It is achieved with the help of Cognitive Radio Networks (CRN).In CRN, the challenging and primary task is to sense the spectrum for usage. Spectrum sensing techniques used with different approaches are analyzed. The framework used in [2] takes the summation of probability of false alarm and probability of missed detection for comparing it with the predefined value for efficient spectrum sensing. The spectrum sensing technique used here is Energy Detection. It overcomes the limitations of conventional energy detection. Multidimensional correlation based technique is used and improves the energy consumption[3].Analyzing the results from the secondary users, cooperative spectrum sensing can be used. For the better detection of spectrum holes, the adaptive window is used with the simultaneous sensing. The author illustrated this with the BER detection and showed that it improves the detection [4]. In CRN, cooperative spectrum sensing can be used to improve the detection. Fusion center collects the binary decision results from the available secondary users and decided where the PU is present in the spectrum or not. In binary decision, 1 represents the PU presence and 0 represents the PU absence in the spectrum. The different decision rule in various fading channels which used in cooperative and decentralized spectrum sensing is analyzed in [5]. The result shows that the proposed system with OR rule provides the better result and AND rule gives the better detection compared to other results. For effective decision, the results with AND rule will be taken for consideration. A different sensing technique quantization based approach is used to get the improved results for the spectrum hole identification. The SU transmits the sensing information in multi bits data form and it is collected by the fusion center to identify the spectrum hole[6]. The minimization function is used to minimize the probability of error in the spectrum. The author used the dual threshold for improving the results by considering the noise uncertainty factor in the signal [7]. The author addressed the problems of performance degradation of the licensed spectrum. Cross layer design can be used to improve the performance of the spectrum. The importance and needs of the cross layer design are produced in [8]. The cross layer based OFDM system is considered for optimizing the utilization of the spectrum. The parameters considered for the optimization are power allocation and the sub carrier assignment [9]. The spectrum is optimized by balancing the power and data rate of the signal to protect the PU from the interference[10].The analysis on cross layer design for spectrum sensing is at the infant stage only. Here, the first two layers are mostly involved in the deigning. The layered approach means it should follow the layered communication from the top to bottom 
layer of OSI model. In cross layer design, the layered communication can be avoided when it is needed for improving the performance of the system. This approach can be used by any layer for its spectrum sensing. While SU is in transmission, PU enters the spectrum then SU has to leave the spectrum and it is in need to identify the other spectrum hole for transmission. In such cases, it moves to the physical layer for getting the spectrum sensing results which involve the data link layer for detection. SU can resume the transmission after getting the results from the physical layer. In the proposed system, OFDM based signal is used. Most of the wireless applications use the OFDM for the communication.The OFDM signal is generated as a first step. Energy detection based spectrum sensing is applied because of the minimum sensing time of an algorithm. Cooperative Decision based sensing is applied with decision rules AND and OR for the effective spectrum sensing.

\section{SYSTEM MODEL}

\subsection{OFDM Signal Generation}

The system used here is OFDM for sensing the primary user signal. The OFDM signal consists of $\mathrm{N}_{\mathrm{m}}$ narrowband signals and sub carriers $\mathrm{L} 1, \mathrm{~L} 2, \ldots . . \mathrm{L}_{\mathrm{Nm}-1}$ as data carriers and $\Delta \mathrm{f}$ is the symbol duration. The signal is generated by passing the narrow band signals $\left(\mathrm{N}_{\mathrm{m}}\right)$ through Inverse Fast Fourier Transform(IFFT).The symbol duration of the OFDM signalis obtained by adding the cyclic prefix to the OFDM signal.The symbol duration is $\mathrm{Ns}=\mathrm{N}_{\mathrm{m}}+\mathrm{N}_{\mathrm{c}}$.

The OFDM base band signal is generated using

$$
Z(t)=\sum_{n=0}^{N t-1} L_{n} \exp (j 2 \pi t n \Delta f)
$$

\subsection{Energy Detection}

The number of CR users considered for the research is $C$. The two hypotheses used for the received signal of PU at the spectrum. The signal of PU received by the $i^{\text {th }} \mathrm{CR}$ user can be represented as

$$
\begin{aligned}
& x(c)_{i}=w(c)_{i}: H 0 \\
& x(c)_{i}=s(c)_{i}+w(c)_{i}: H 1
\end{aligned}
$$

where $c=1,2, \ldots \mathrm{T}$, where $\mathrm{T}$ is the number of samples taken for generating the received signal $\mathrm{x}(\mathrm{c})$ at the $\mathrm{i}^{\text {th }} \mathrm{CR}$ user and $\mathrm{w}(\mathrm{c})$ is the Additive White Gaussian Noise (AWGN) at the $i^{\text {th }}$ user. The received signal for the given signal to noise ratio is represented as $\mathrm{s}(\mathrm{c})$. The presence and absence of the PU signal is represented as $\mathrm{H}_{0}$ and $\mathrm{H}_{1}$.
The simple technique,energy detection is used for the detection along with the OFDM signal. Energy of the received signal is calculated as

$$
\operatorname{Energy}_{i}=\sum_{1}^{T}\left(x(c)_{i}\right)^{2}
$$

The energy is compared with the predefined threshold using the following equation.

$$
\begin{aligned}
& \operatorname{Energy}(i)<\lambda(i) \\
& \operatorname{Energy}(i)>\lambda(i)
\end{aligned}
$$

\subsection{Cooperative Spectrum sensing (CSS)}

The advantage of CSS utilizes the advantages of avoiding selective fading channel problems. In CSS, either Hard Decision and Soft Decision rule can be used. In this system, the hard decision rule is used. Hard Decision rule like AND, OR used in this system. The AND rule decides the presence by comparing all the binary decisions provided by the CR users. If all the CR users provide the decision as 1 then it is taken as presence of $\mathrm{PU}$ in the spectrum otherwise it is considered as absence.OR rule means if any one user provides the presence then it is considered as presence. The effective rule is AND rule for effectively detecting the presence of the user in the spectrum. The formula for deciding the PU presence in the spectrum by the fusion center is

$$
\begin{aligned}
& P_{d, A N D}=\left(\overline{P_{d, i}}\right)^{C} \\
& P_{f, A N D}=\left(P_{d, i}\right)^{C} \\
& P_{d, O R}=1-\left(1-\overline{P_{d, i}}\right)^{C} \\
& P_{f, O R}=1-\left(1-P_{d, i}\right)^{C}
\end{aligned}
$$

In this method, the hard decision rule is applied on the energy detection with OFDM signals.Here, OFDM is considered for spectrum sensing and Energy detection is applied to determine the spectrum effectively.

\subsection{Cross Layer Design with CSS}

Cross Layer design with Cooperative spectrum sensing is applied for the efficient detection of the signal.Spectrum sensing is done at the physical Layer only. Here both the Data Link Layer and Physical Layer of OSI layer is used for efficient detection.The cross layer framework for spectrum sensing can be described as in Figure 1.Cross Layer Design is proposed here . In Data Link Layer, Power of the OFDM signal is generated. The power is used for deciding the presence of the PU in the spectrum which is done at the physical layer.OFDM signal energy is applied with the energy detection method for generating the OFDM signal . 


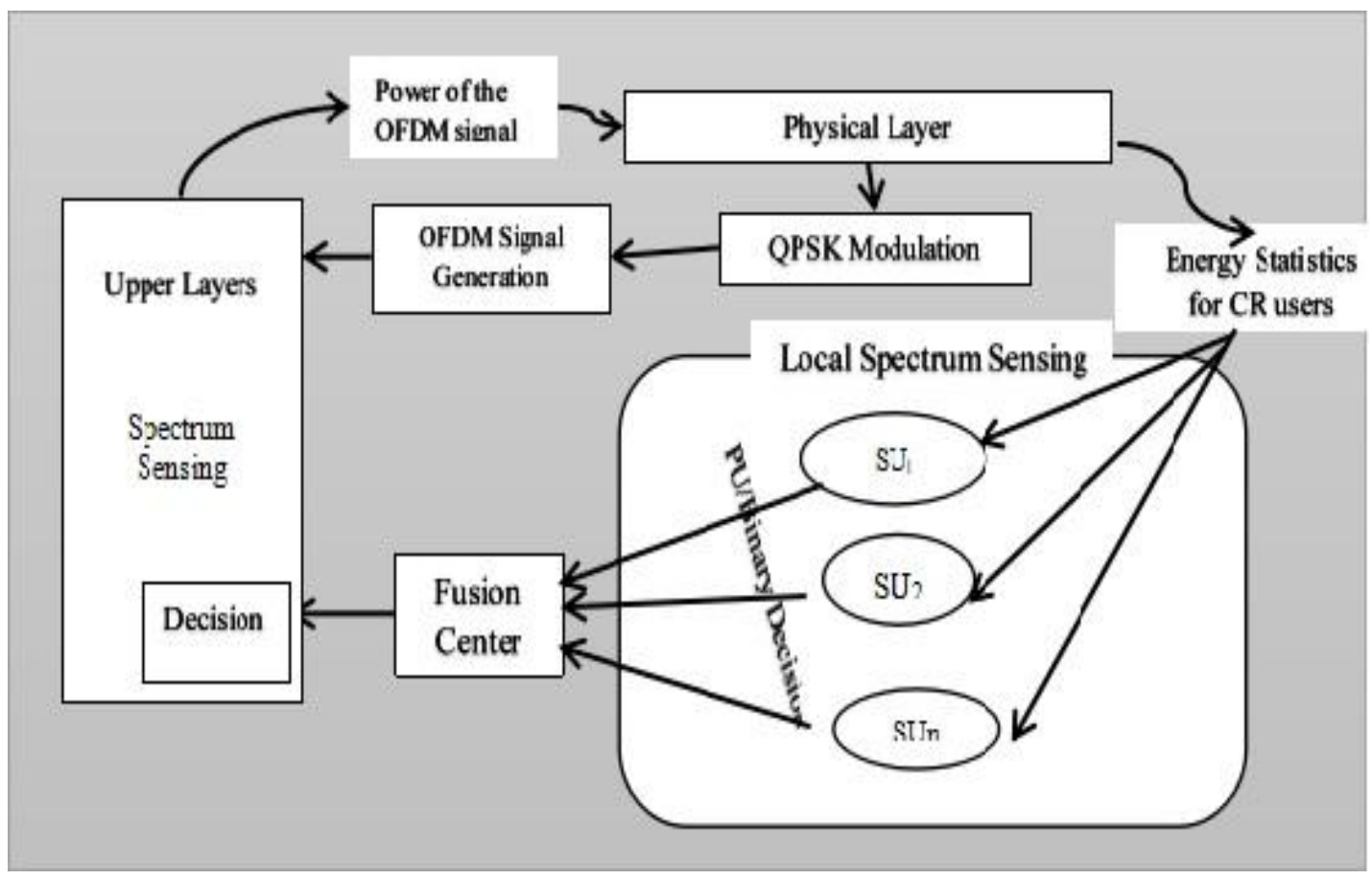

Figure 1: Proposed Cross Layer Framework

\section{THE PROPOSED SYSTEM}

The random noise added to the OFDM generated signal as in equation 1 . Then the transmitted signal becomes

$$
\operatorname{signal}_{k}(t)=Z_{k}(t)+h_{k}(t)
$$

Where $\mathrm{k}$ represents the total number of OFDM symbols generated based on the number of narrow band signals and number of sub carriers. The power of the signal is generated based on the total number symbols generated. It is determined using the linear SNR.The power of the one OFDM sub carrier is obtained using

$$
\operatorname{power}_{k}(t)=\sum_{1}^{k}\left(\operatorname{signal}_{k}(t)\right)^{2} / k
$$

Cross layer design is applied by moving from the second layer of OSI to the first layer of OSI for detecting the signal energy with the help of linear SNR. Energy Detection signal is determined using hypothesis 1 and 2 . We are assuming that only the power of the OFDM signal is known. The received signal at the $\mathrm{i}^{\text {th }} \mathrm{CR}$ user is

$$
y(t)_{i}=\left(\sqrt{\text { snr }^{*} \text { power }_{k}(t)}\right)+\text { noise }
$$

The decision of the PU detection at the spectrum is based on the following equations.

$$
\begin{array}{ll}
D\left(y(t)_{i}\right)=1 / T\left(y(t)_{i}^{*} w(t)_{i}\right)>\lambda & \text { Under } H_{I} \\
D\left(y(t)_{i}\right)=1 / T\left(y(t)_{i} * w(t)_{i}\right)<\lambda & \text { Under } H_{0}
\end{array}
$$

The energy of the signal decides the PU detection.

$$
E_{i}=1 / T * \sum_{1}^{T-1}\left(y(t)_{i}\right)^{2}
$$

The primary user presence is decided based on the following hypothesis.

$$
\begin{array}{ll}
E_{i}>\lambda & \text { PU is Present } \\
E_{i}<\lambda & \text { PU is Absent }
\end{array}
$$

The probability of false alarm $P_{f}$ and the probability of detection $P_{d}$ is calculated as in [11].T is the number of samples, $\mathrm{E}_{\mathrm{i}}$ is the energy statistics for comparing with the threshold $\lambda$, $\sigma_{\omega}$ is the noise variance of the signal and $\sigma_{s}$ is the power of the generated signal. 


$$
\begin{gathered}
P_{f}=\operatorname{Pr}\left(E_{i}<\lambda: H 0\right) \\
P d=\operatorname{Pr}\left(E_{i}>\lambda: H 1\right) \\
P_{f}=Q\left(\frac{\lambda-T \sigma_{\omega}^{2}}{\sqrt{2 T \sigma_{\omega}^{4}}}\right) \\
P_{d}=Q\left(\frac{\lambda-T\left(\sigma_{s}{ }^{2}+\sigma_{\omega}{ }^{2}\right)}{\sqrt{2 T\left(\sigma_{s}{ }^{2}+\sigma_{\omega}{ }^{4}\right.}}\right) \\
\lambda=\sigma_{\omega}{ }^{2}\left(Q^{-1}\left(P_{f}\right) \sqrt{2 T}+T\right)
\end{gathered}
$$

The obtained Pd for the samples of each CR user is sent to the fusion center for deciding the presence of the signal. Based on the Hard Decision rule AND,OR , the decision is made. For effective decision AND rule is used by the Fusion center for deciding the presence. In the proposed system $P_{f}$ values from 0.01 to 1.00 with the increasing 0.01 at each step for finding the probability of detection at each CR user is used.

CSS is applied with the cross layer design for the efficient detection. The Hard decision rule is applied to find the performance of the proposed system. The decision rules AND, OR are used for the comparison with the other existing systems. Figure 3 and Figure 4 describes the result of the produced system for the SNR $-18 \mathrm{~dB}$ and $-15 \mathrm{~dB}$ respectively. The figures are for the cross layer with fusion rules AND and OR. The proposed system produces the higher results than the existing system. There are many authors used the cooperative spectrum sensing with hard decision rules. Compared with the other systems, the proposed one detects the user at the spectrum with more accuracy rate in low SNR values.

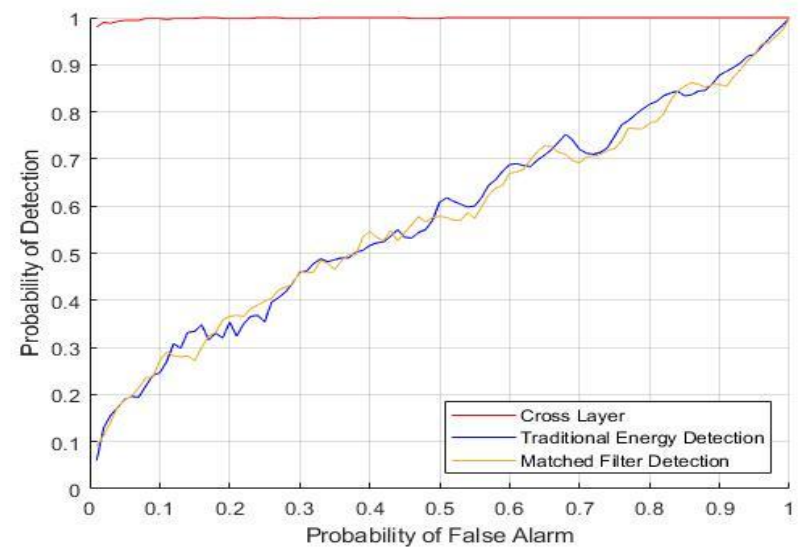

Figure 2 : Probability of Detection vs Probability of False Alarm for $\mathrm{N}=150, \mathrm{SNR}=-15 \mathrm{~dB}$ and $\mathrm{CR}$ users $=10$ of Cross Layer Design and Traditional Spectrum Sensing Techniques

\section{RESULTS AND DISCUSSION}

The proposed system results are analyzed in the terms of probability of detection of the user at the spectrum. The sample taken for analysis is the important parameter for the detection. Number of samples can be chosen as any positive integer.Nowadays, the large number of communication are taken place among the users. The minimum number of samples taken for analysis are 20,40,60,80,100 and 150. The system produces the high accuracy results when the number of samples number taken for analysis in large. The number of CR users taken is 10. First step, OFDM signal is generated. The inputs for the OFDM signal generation are the number of sub carriers, cyclic prefix length to mitigate the interference, the number of data carriers, the frame size and the number of inputs taken for signal generation. Figure 2 is the compared results of the cross layer design, traditional energy detection and matched filter detection. Most analysis are done with the help of matched filter analysis and energy detection. So these two are compared with the proposed cross layer spectrum sensing technique. The cross layer design produces the $100 \%$ detection for the SNR above $-15 \mathrm{~dB}$.

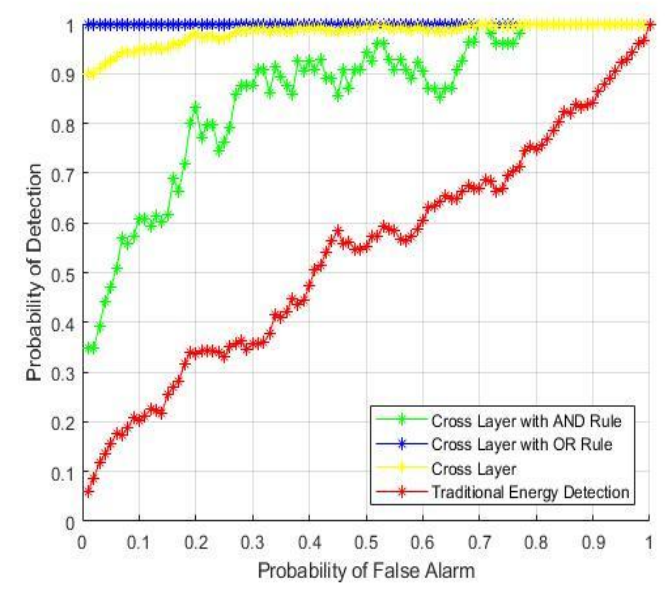

Figure 3 : Probability of Detection vs Probability of False Alarm for $\mathrm{N}=150, \mathrm{SNR}=-\mathbf{1 8} \mathrm{dB}$ and $\mathrm{CR}$ users $=10$

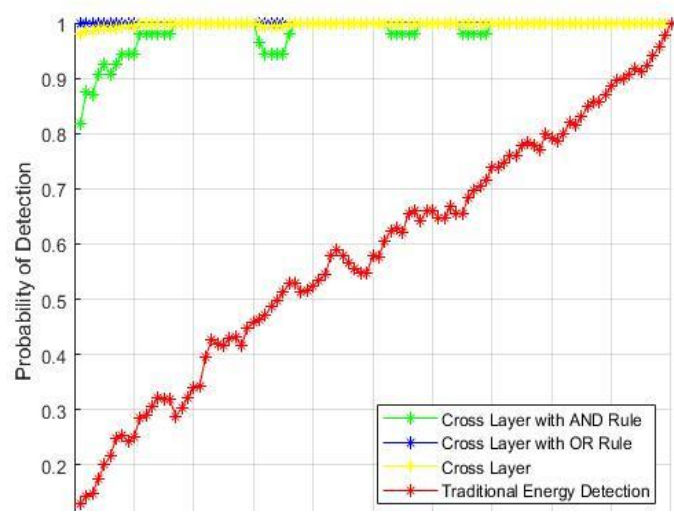

Figure 4 : Probability of Detection vs Probability of False Alarm for $\mathrm{N}=150, \mathrm{SNR}=-15 \mathrm{~dB}$ and $\mathrm{CR}$ users $=10$ 
The proposed cross layer frame work results are illustrated in Figure 5. The results are displayed for the different number of samples. If the size of the sample is increased then the result of the proposed system is also increased. It produces the higher results compared with the existing system for the low SNR values. From that, the cross layered architecture produces the high results than the layered architecture.

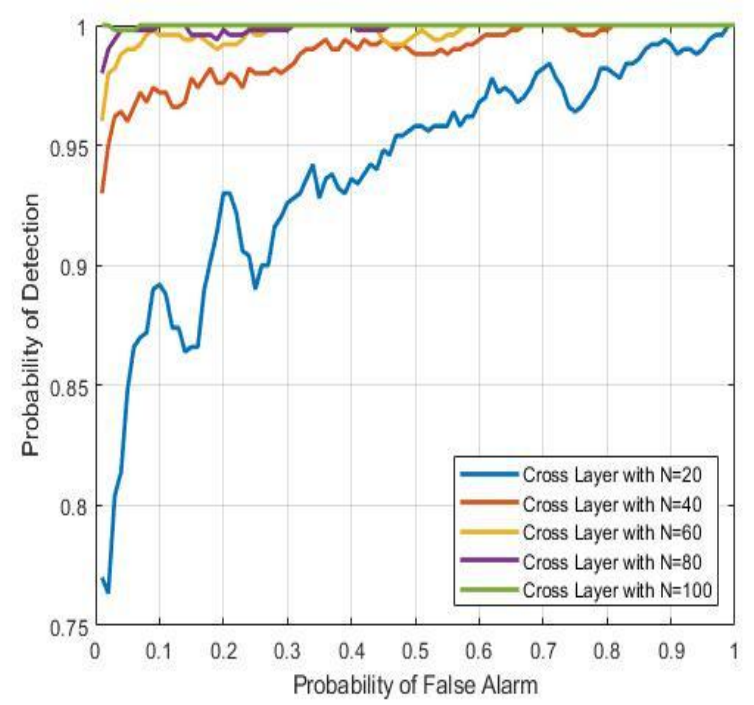

Figure 5 : Probability of Detection vs Probability of False Alarm for Different number samples and SNR= $-10 \mathrm{~dB}$ of a proposed Cross Layer Design

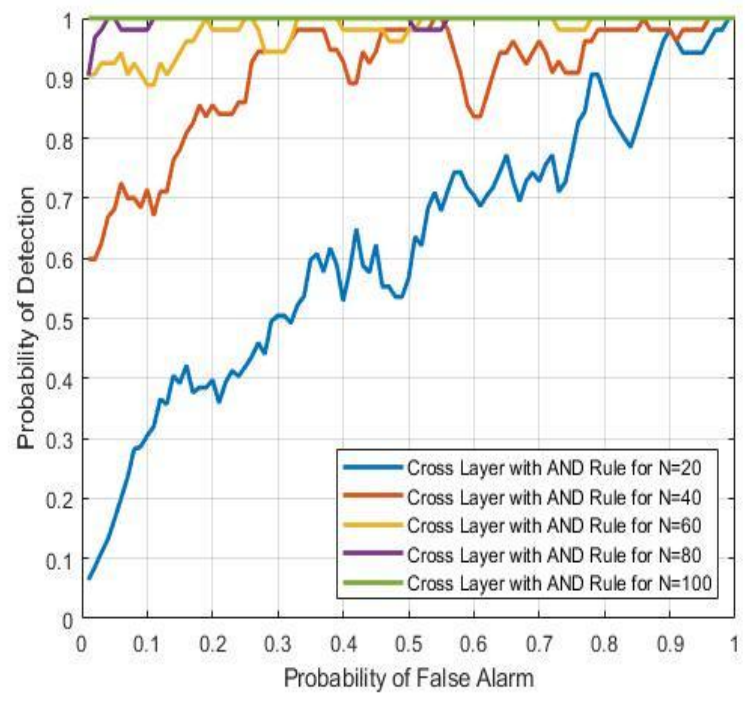

Figure 6 : Probability of Detection vs Probability of False Alarm for Different number samples and $S N R=-10 \mathrm{~dB}$ of a proposed Cross Layer Design with AND Decision Rule.
In today's wireless world, there are more number of CR users in need of accessing the spectrum due to scarcity. The coordination among all the CR users must exist for improving the spectrum utilization. CSS with different rules are applied to get the better results. Mitigating the interference is the important task to be achieved by the CR technology for effective utilization. The AND rule results may be considered for the betterment of the system. Cross layer Design with AND rule results for the different samples are plotted in Figure 6.

\section{CONCLUSION}

Cross Layer Design with Cooperative Spectrum Sensing is studied and implemented in the proposed system. Cross Layer is implemented by including the layers of physical layer, data link layer and higher layers. The performance of the system is achieved with the help of OFDM and Energy detection technique. Cooperative spectrum sensing with AND and OR rule is applied with the cross layer approach. However the better results are produced by OR decision rule, the cooperative spectrum sensing with AND rule is the best technique to avoid the interference used by Fusion Center. The results of the proposed work is simulated and it shows that it outperforms the other existing system. As a future study, the cross layer design with soft decision rule and dynamic threshold for the energy detection to be considered for providing the better solution to the spectrum scarcity.

\section{REFERENCES}

[1] P. Kolodzy:Spectrum policy task force,Rep. ET Docket 02-135, Federal Communications Commission, Washington, DC, USA, 2002.

[2] Hu, X.-L., Ho, P.-H. and Peng L:Fundamental Limitations in Energy Detection for Spectrum Sensing. J. Sens. Actuator Netw 2018; 7-25.

[3] Dongyue Xue, Eylem Ekici, and Mehmet C. Vura.Cooperative Spectrum Sensing in Cognitive Radio Networks Using Multidimensional Correlations.IEEE Transactions on Wireless Communications 2014;13

[4] Lu, Y., Wang, D. \& Fattouche, M. Cooperative spectrumsensing algorithm in cognitive radio by simultaneous sensing and BER measurements. J Wireless Com Network 2016;136

[5] Ashish Rauniyar, Jae Min Jang, and Soo Young Shin.Optimal Hard Decision Fusion Rule for Centralized and Decentralized Cooperative Spectrum Sensing in 
International Journal of Engineering Research and Technology. ISSN 0974-3154, Volume 13, Number 6 (2020), pp. 1095-1100

(C) International Research Publication House. https://dx.doi.org/10.37624/IJERT/13.6.2020.1095-1100

Cognitive Radio Networks, Journal of Advances in Computer Networks 2015; 3

[6] Fu, Y.,Yang, F.He, Z. A Quantization-Based Multibit Data Fusion Scheme for Cooperative Spectrum Sensing in Cognitive Radio Networks. Sensors 2018,;8,:473.

[7] Wan, R., Ding, L., Xiong, N. et al. Dynamic dual threshold cooperative spectrum sensing for cognitive radio under noise power uncertainty. Hum. Cent. Comput. Inf. Sci. $2019 ; 9: 22$

[8] Garg, Piyush. A Survey of Cross-Layer Design in Cognitive Radio Networks: Issues and Proposed Solutions 2017: 10.13140/RG.2.2.19618.73920.

[9] R. Wang, V. K. N. Lau, L. Lv and B. Chen.Joint crosslayer scheduling and spectrum sensing for OFDMA cognitive radio systems. IEEE Transactions on Wireless Communications 2017; 8: 2410-2416

[10] Zhang, L., Zhuo, F. \& Xu, H. A cross-layer optimization framework for congestion and power control in cognitive radio ad hoc networks under predictable contact..Journal of Wireless Communications and Networking 2018;57

[11] D. Cabric, A. Tkachenko and R. W. Brodersen.Experimental Study of Spectrum Sensing based on Energy Detection and Network Cooperation.proceedings of the First International Workshop on Technology and Policy for Accessing Spectrum (TAPAS 2006)August 2006.

[12] https://www.yumpu.com/en/document/read/43694940/cr oss-layer-design-of-cognitive-radio-mb-ofdm-systemsutm. 